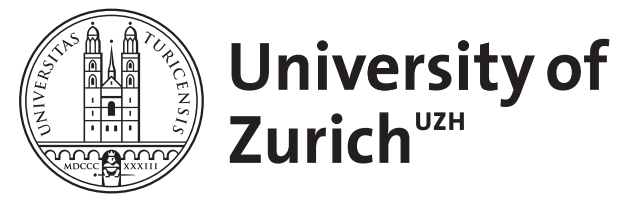

\title{
Medical algorithm: Diagnosis and treatment of eosinophilic esophagitis in adults
}

\author{
Greuter, Thomas ; Straumann, Alex
}

DOI: https://doi.org/10.1111/all.14112

Posted at the Zurich Open Repository and Archive, University of Zurich ZORA URL: https://doi.org/10.5167/uzh-181125

Journal Article

Accepted Version

Originally published at:

Greuter, Thomas; Straumann, Alex (2020). Medical algorithm: Diagnosis and treatment of eosinophilic esophagitis in adults. Allergy, 75(3):727-730.

DOI: https://doi.org/10.1111/all.14112 


\title{
MEDICAL ALGORITHM: Diagnosis and Treatment of Eosinophilic Esophagitis in
}

\section{Adults}

\author{
Re-Submission to Allergy
}

Version 2019-10-12

Thomas Greuter MD and Alex Straumann MD

Swiss EoE Clinic, Department of Gastroenterology and Hepatology, University Hospital Zurich, Zurich Switzerland

\section{Address for Correspondence}

Thomas Greuter, M.D., Department of Gastroenterology and Hepatology, University Hospital Zurich, Raemistrasse 100,CH-8091 Zurich, Switzerland, E-mail: Thomas.Greuter@usz.ch

Word count: 595 words (max. 600 words)

\section{ACKNOWLEDGEMENTS}

Grant support: This work was supported by a grant from the Swiss National Science Foundation to AS (grant no. 32003B_160115) and TG (grant no. P2ZHP3_168561), a young investigator award from the Swiss Society of Gastroenterology to TG, a training grant from Consortium of Eosinophilic Gastrointestinal Disease Researchers (CEGIR) to TG. CEGIR (U54 Al117804) is part of the Rare Disease Clinical Research Network (RDCRN), an initiative of the Office of Rare Diseases Research (ORDR), NCATS, and is funded through collaboration between NIAID, NIDDK, and NCATS. CEGIR is also supported by patient advocacy groups including American Partnership for Eosinophilic Disorders (APFED), Campaign Urging Research for Eosinophilic Diseases (CURED) and Eosinophilic Family Coalition (EFC)

Potential competing interests: AS has consultant contracts with Celgene-Receptos, Falk Pharma, Sanofi-Regeneron and Tillots. TG received a travel grant from Falk Pharma GmbH and Vifor, has a consultant contract with Sanofi-Aventis and received an unrestricted research grant from Novartis 


\section{INTRODUCTION}

Eosinophilic esophagitis (EOE) is a chronic-inflammatory disease of the esophagus, defined clinically by presence of symptoms of esophageal dysfunction and histologically by an eosinophil predominant infiltration in the esophageal mucosa. ${ }^{1}$ Due to its chronicity, EoE requires both short-term and long-term treatment. ${ }^{2}$ Therapeutic goals are: 1) to control clinical activity (resolution of symptoms); and 2) to control biological activity to avoid stricture formation and bolus impaction, a potentially life-threatening event. Proper diagnosis of EoE and successful treatment are key in EoE management.

\section{DIAGNOSIS}

A thorough medical history is the first diagnostic step (Figure 1). Typical symptoms of esophageal dysfunction such as solid-food dysphagia and/or recurrent bolus impactions in young patients with allergic comorbidities considerably increase the pre-test probability for EoE. To prove suspected EoE, esophagogastroduodenoscopy with biopsies taken from the distal and proximal esophagus is needed. ${ }^{3}$ Multiple biopsies increase diagnostic accuracy. ${ }^{4}$ At least 6 biopsies ( 3 from the proximal and 3 from the distal esophagus) should be taken. Typical clinical presentation together with eosophageal eosinophilia is sufficient to diagnose EoE. However, in case of non-typical symptoms or unusual endoscopic findings, other conditions leading to an eosinophilic infiltration should be considered such as reflux disease (reflux symptoms, signs of reflux esophagitis and/or hiatal hernia) or eosinophilic gastroenteritis (atypical symptoms such as dyspepsia, vomiting, diarrhea, endoscopic abnormalities in stomach or duodenum).

Lately, it has become apparent that some patients can present with an EoE-like disease, defined as typical clinical presentation, but lack of significant eosinophilic infiltration in the esophagus. ${ }^{5}$ In these cases, EoE under treatment (PPI intake) and motility disorders have to be considered also. Review of histology slides by an expert EoE pathologist and immunohistochemical analyses are recommended to capture subtle histological changes such as basal zone hyperplasia or spongiosis and cellular infiltrates different from eosinophils consistent with EoE-like disease. In unclear cases, further work-up with barium swallow and high-resolution manometry should be performed. 
In recent years, new diagnostic tools such as the Endoflip, esophageal string test and cytosponge have been studied. However, their exact role in the diagnostic algorithm have not been clearly defined and as of yet their routine use cannot be recommended. ${ }^{2}$

\section{TREATMENT}

Treatment of EoE consists of the 3 D's: Drugs, Diet and Dilation. ${ }^{2}$ Dilation should be seen as an add-on treatment since it does not affect underlying inflammation (Figure 2). Two drug categories are available: Swallowed topical corticosteroids (STC) and PPI. Both should be used in high dose at the beginning. The most frequently applied dietary strategies are the 6food elimination diet (FED) with a potential step-down over time and the 2-4-6 FED step-up approach. ${ }^{6,7}$ In case of severe disease (frequent bolus impactions, stricturing phenotype), we recommend to use the most potent therapeutic agent, which are high dose STC (either budesonide $2 \times 1 \mathrm{mg}$ or fluticasone $2 \times 880 \mathrm{ug}$ or fluticasone $2 \times 1 \mathrm{mg}$ if available). ${ }^{8}$ High-dose treatment should be continued for 6 weeks. In case of non-response or only partial response, expanding the induction phase to 12 weeks has been shown to be of further benefit. ${ }^{9}$

Response to treatment should be assessed in every patient, which includes resolution of symptoms, endoscopic signs of inflammation and esophageal eosinophilia (= complete remission). In case of remission, PPI and STC doses can be lowered, although an optimal maintenance dose has yet to be determined. Based on recently presented data, half the induction dose appears to be a reasonable approach. ${ }^{10}$ If remission was achieved with empiric elimination diet, re-introduction of food should be initiated. Most importantly, any therapy should be maintained as cessation of treatment results in an EoE relapse within only few months. ${ }^{11}$ If histological response is not achieved, we recommend switching from PPI ("PPI non-response") or elimination diet to STC. In case of less restrictive empiric elimination diets, a step-up approach can be taken alternatively (such as the 2-4-6 elimination diet strategy). If STC have failed to induce histological remission, a dietary approach or PPI high dose treatment might be considered. In addition, compliance and handling should be assessed as incorrect administration is the leading cause for refractory disease. If EoE remains active for another 8 to 12 weeks, pseudo-refractory EoE should be ruled out (Supplementary Table 1). In case of true refractory disease, the monoclonal IL4-receptor antibody Dupilumab might be considered, although it has not been approved yet for the management of EoE. ${ }^{12}$ 
If a patient shows a histological and endoscopic response, but remains symptomatic, we recommend endoscopic dilation. Stricturing disease can be easily missed during esophagogastrodudoenscopy. ${ }^{13}$ Dilation may be repeated after few weeks up to an esophageal diameter of $16-18 \mathrm{~mm}$. Dilation should always be done with caution and individually tailored on a case-by-case basis in order to minimize the risk of procedure-induced perforation.

\section{LEGENDS}

Figure 1: Algorithm for the diagnosis of eosinophilic esophagitis in adults. EGD, esophagogastroduodenoscopy; hpf; high-power field; peak eos count, peak eosinophil count in the esophageal mucosa

Figure 2: Algorithm for the treatment of eosinophilic esophagitis in adults. PPI, proton-pump inhibitors; STC, swallowed topical corticosteroids

Supplementary Table 1: Causes for pseudo-refractory EoE.

\section{REFERENCES}

1. Liacouras CA, Furuta GT, Hirano I, et al. Eosinophilic esophagitis: updated consensus recommendations for children and adults. J Allergy Clin Immunol. 2011;128(1):3-20.e26; quiz 21-22.

2. Greuter T, Alexander JA, Straumann A, Katzka DA. Diagnostic and Therapeutic Long-term Management of Eosinophilic Esophagitis- Current Concepts and Perspectives for Steroid Use. Clin Trans/ Gastroenterol. 2018;9(12):e212.

3. Dellon ES, Liacouras CA, Molina-Infante J, et al. Updated International Consensus Diagnostic Criteria for Eosinophilic Esophagitis: Proceedings of the AGREE Conference. Gastroenterology. 2018;155(4):1022-1033.e1010.

4. Gonsalves N, Policarpio-Nicolas M, Zhang Q, Rao MS, Hirano I. Histopathologic variability and endoscopic correlates in adults with eosinophilic esophagitis. Gastrointest Endosc. 2006;64(3):313319.

5. Straumann A, Blanchard C, Radonjic-Hoesli S, et al. A new eosinophilic esophagitis (EoE)-like disease without tissue eosinophilia found in EoE families. Allergy. 2016;71(6):889-900.

6. Kagalwalla AF, Sentongo TA, Ritz S, et al. Effect of six-food elimination diet on clinical and histologic outcomes in eosinophilic esophagitis. Clin Gastroenterol Hepatol. 2006;4(9):1097-1102.

7. Molina-Infante J, Arias Á, Alcedo J, et al. Step-up empiric elimination diet for pediatric and adult eosinophilic esophagitis: The 2-4-6 study. J Allergy Clin Immunol. 2018;141(4):1365-1372.

8. Dellon ES, Woosley JT, Arrington A, et al. Efficacy of Budesonide vs Fluticasone for Initial Treatment of Eosinophilic Esophagitis in a Randomized Controlled Trial. Gastroenterology. 2019;157(1):65-73.e65.

9. Lucendo AJ, Miehlke S, Schlag C, et al. Efficacy of Budesonide Orodispersible Tablets as Induction Therapy for Eosinophilic Esophagitis in a Randomized Placebo-Controlled Trial. Gastroenterology. 2019;157(1):74-86.e15.

10. Lucendo A, Miehlke S, Vieth M, et al. Budesonide orodispersable tablets are highly effective to maintain clinico-histological remission in adult patients with eosinophilic esophagitis: Results from the 48-weeks, double-blind, placebo-controlled, pivotal EOS-2 trial. Gastroenterology. 2019; 156(6, Suppl. 1):S-1509.

11. Greuter T, Bussmann C, Safroneeva E, et al. Long-Term Treatment of Eosinophilic Esophagitis With Swallowed Topical Corticosteroids: Development and Evaluation of a Therapeutic Concept. Am J Gastroenterol. 2017;112(10):1527-1535. 
12. Hirano I, Dellon E, Hamilton J, et al. Efficacy of Dupilumab in a Phase 2 Randomized Trial of Adults With Active Eosinophilic Esophagitis.Gastroenterology. In press, 2019. In.

13. Podboy A, Katzka DA, Enders F, et al. Oesophageal narrowing on barium oesophagram is more common in adult patients with eosinophilic oesophagitis than PPI-responsive oesophageal eosinophilia. Aliment Pharmacol Ther. 2016;43(11):1168-1177. 